\title{
Prognostication and monitoring of mesothelioma using biomarkers: a systematic review
}

\author{
David T Arnold ${ }^{\star}, 1$, Duneesha De Fonseka ${ }^{1}$, Fergus W Hamilton ${ }^{2}$, Najib M Rahman ${ }^{3}$ and Nick A Maskell ${ }^{1}$ \\ ${ }^{1}$ Academic Respiratory Unit, School of Clinical Sciences, University of Bristol, Southmead Hospital, Learning and Research Centre, \\ Westbury-on-Trym, Bristol BS10 5NB, UK; ${ }^{2}$ Southmead Hospital, North Bristol NHS Trust, Bristol BS10 5NB, UK and ${ }^{3}$ Oxford \\ Centre for Respiratory Medicine and Oxford NIHR Biomedical Research Centre, Churchill Hospital, Oxford OX3 7LE, UK
}

Background: Radiological markers of treatment response and prognostication in malignant pleural mesothelioma have limitations due to the morphology of the disease. Serum or pleural fluid biomarkers that could act as an adjunct to radiological assessment would be of significant value. The aim of this review was to collate and summarise the literature relating to this topic.

Methods: A systematic review was performed on the databases Pubmed and EMBASE to identify relevant studies. Two independent researchers read the abstracts and used the Quality in Prognostic Studies tool to assess the quality of the evidence.

Results: Forty-five studies were identified from the current literature. Twenty studies investigated the role of serum soluble mesothelin with majority suggesting that it has variable utility as a baseline test but when measured serially correlates with treatment response and prognosis. Several studies demonstrated that serum osteopontin correlated with survival at baseline. Other biomarkers have shown prognostic utility in individual studies but are yet to be reproduced in large cohort studies.

Conclusions: From the available literature no serum or pleural fluid biomarker was identified that could be recommended currently for routine clinical practice. However, a falling serum soluble mesothelin might correlate with treatment response and improved survival.

Malignant pleural mesothelioma (MPM) is a rapidly progressive and invariably fatal malignancy. Mean survival is 9-14 months from diagnosis (Chapman et al, 2008). First-line palliative chemotherapy with pemetrexed and a platinum-based agent (Cisplatin or Carboplatin) has been the standard of care for over a decade (Vogelzang et al, 2003). However, following encouraging results from the MAPS trial, many guidelines are now advocating the addition of bevacizumab to this regimen (Zalcman et al, 2016). Despite this, chemotherapy has only a modest impact on survival of around 2-3 months with a possible small improvement in symptomatology (Arnold et al, 2015). Response to chemotherapy differs greatly between patients with a partial response rate of 30-40\% (Vogelzang et al, 2003). Clinical trials and clinicians use radiological markers to assess treatment response and progressionfree survival (PFS). The current best practice is serial thoracic computerised tomography (CT) scans reported using the modified RECIST criteria, a technique which only partially allows for the fact that MPM usually grows as a pleural rind as opposed to a spherical mass. Other challenges, such as the presence of pleural fluid (pf) and benign asbestos-related plaques, make radiological assessment of MPM difficult (Armato et al, 2006).

A blood or pf biomarker that could act as an adjunct to radiological assessment by giving prognostic information as well as reflecting response to treatment would be of considerable use to clinicians. The majority of literature on biomarkers in MPM focuses on their utility as a screening or diagnostic test. Most

*Correspondence: Dr DT Arnold; E-mail: arnold.dta@gmail.com

Received 18 October 2016; revised 2 January 2017; accepted 11 January 2017; published online 7 February 2017

(C) 2017 Cancer Research UK. All rights reserved 0007-0920/17 
researched is soluble mesothelin (SM)-related peptide often called SM. Soluble mesothelin is the circulating form of a $40 \mathrm{kDa}$ membrane-bound glycoprotein and is highly expressed by mesothelial cells in MPM and some other cancers (Robinson et al, 2003). Soluble mesothelin levels are much higher in epithelioid MPM compared with other histological subtypes, and in larger tumours. However, its diagnostic ability in both serum and pf is limited by an inability to exclude MPM with a negative result. Another biomarker is megakaryocyte potentiating factor (MPF), also called N-ERC/mesothelin as it is formed from the same precursor protein as SM (Hollevoet et al, 2010). Osteopontin (OPN) is a glycoprotein that mediates cell-matrix interactions and has been shown to infer a poor phenotype when raised in other malignancies, including breast, lung and colon (Pass et al, 2005; Shojaei et al, 2012). Finally, fibulin-3 is an extracellular glycoprotein, which has shown promise in the diagnosis of MPM (Pass et al, 2012), but information on its role in prognostication is limited. We performed a systematic review of studies that had assessed the role of biomarkers in providing prognostic and treatment response information for MPM in an attempt to guide clinicians as to the strength of evidence for use in current practice.

\section{MATERIALS AND METHODS}

Search strategy. The databases PubMed (Medline) and EMBASE were interrogated for papers related to our study question. The 30 June 2016 was used as a cut-off with no early limit date applied. The search was limited to English language papers using the search terms shown in Supplementary Table 1. The search terms were designed to limit the search to papers that provided information on prognostication and disease monitoring and have been used in previous such studies (Altman, 2001; Dretzke et al, 2014). Articles were also identified using the 'related articles' function of PubMed and the references of the selected papers were assessed for other relevant papers. Two reviewers (DA and $\mathrm{FH}$ ) screened the abstracts for study eligibility; any disagreements were resolved by mutual consensus.

\section{Study inclusion criteria}

- Involved the measurement of a serum or pf biomarker in patients with proven MPM.

- Treatment response or survival data collected and correlated with biomarkers.

\section{Study exclusion criteria}

- Reported only biomarkers from tumour immunohistochemistry.

- Results duplicated from another selected study.

- Involved less than 10 patients with MPM.

- Conference abstract or letter.

Quality assessment and data extraction. Once the full set of articles were extracted, two reviewers (DA and $\mathrm{FH}$ ) independently applied the quality in prognostic studies (QUIPS) tool to assess the quality of selected studies (Hayden et al, 2013). The QUIPS score of each paper has been reported in Supplementary Table 2. In addition, routine data were extracted from the studies including author, publication year, study type (prospective or retrospective), patient number, histological subtype, patient treatment and biomarkers studied.

Data extraction was dependant on the findings reported by the individual studies. Correlation of biomarkers with survival is reported using univariate or multivariate Cox-regression analysis unless otherwise stated. Any survival time comparisons are reported using hazard ratios (HR) with 95\% confidence intervals (CI).

\section{RESULTS}

The search strategy generated 1325 abstracts from the Pubmed and EMBASE databases. After screening all the abstracts, 68 were read in full by the independent reviewers. A further 23 were excluded as they did not meet the review criterion (see Figure 1). Therefore, 45 studies were included in the final review. Because of considerable heterogeneity between studies in areas such as histological subtype, biomarker testing and patient therapy, no attempt to combine or meta-analyse the data was made. The selected studies are shown in Table 1 and summarised by biomarker below.

Soluble mesothelin (SM). Serum/plasma SM was the most studied biomarker with 20 studies (18 prospective, 2 retrospective), a total of 1578 patients, investigating its role as a marker of prognostication or treatment response (Robinson et al, 2003; Cristaudo et al, 2007; Grigoriu et al, 2007; Pass et al, 2008; Schneider et al, 2008; Grigoriu et al, 2009; Wheatley-Price et al, 2010; Creaney et al, 2011; Hollevoet et al, 2011; Yamada et al, 2011; Franko et al, 2012; Hollevoet et al, 2012; Kao et al, 2012; Creaney et al, 2013; Nowak et al, 2013; Creaney et al, 2014; Hassan et al, 2014; Linch et al, 2014; Hooper et al, 2015; Pass et al, 2016). Early studies were primarily aimed at SM's ability to diagnose MPM from other malignant or benign lung pathologies with its role as a prognostic indicator a secondary outcome. The earliest study of SM in prognosis, correlated tumour size on CT with baseline SM, finding that levels were significantly higher in larger tumours $(P<0.01)$ and those of epithelioid histology $(P<0.01)$, but there was no correlation with overall survival (OS) (Robinson et al, 2003). Studies by Cristaudo et al (2007) and Grigoriu et al (2007) using the Mesomark ELISA found that higher baseline SM was correlated with worse OS using cut-offs of $1 \mathrm{nmoll}^{-1}$ (HR: 1.6, CI: 1.1-2.4) and $3.5 \mathrm{nmoll}^{-1}$ (HR: 2.8, CI: 1.4-5.6), respectively. Both papers combined patients who were treated with surgery, chemotherapy or best supportive care (BSC). The cut-offs used were selected from diagnostic studies or maximisation of HR models. In a study of 91 MPM patients who received a variety of chemotherapeutic regimens, there was a significant difference in OS between low $\left(<3.5 \mathrm{nmoll}^{-1}\right)$ and high baseline SM levels of 17.1 months vs 8.4 months, respectively (Schneider et al, 2008). This relationship was statistically significant at multivariate analysis (HR: 1.9, CI: $1.1-3.5, P=0.025$ ) but lost statistical significance when applied to epithelioid histology alone. Two studies from Creaney et al $(2013,2014)$ involving 96 and 82 patients, respectively, found no correlation between OS and baseline serum SM.

An earlier prospective study from the same author tested the role of serum SM as a proxy for treatment response when measured serially (Creaney et al, 2011). They recruited 95 patients with MPM and tested serum SM at baseline, every 3 months and before every chemotherapy cycle, alongside thoracic CT scanning. Baseline SM was not correlated with OS at multivariate analysis when radiological markers of FDG-PET were included. In the chemotherapy group $(n=61)$, there was a correlation between response on modified RECIST CT scans and changes in SM $(P=0.023)$. They classified a rise or fall in SM as a change of greater than $25 \%$, otherwise classifying as stable SM. In patients with partial response on CT (17 out of 55) none had a rise in SM with 5 stable levels and 12 falling. In 28 patients who had a repeat FDG-PET as part of follow-up, there was a correlation between percentage change in SM levels and percentage change in tumour TGV and volume $(P<0.01)$. They also found a correlation between change in SM levels and OS with both the stable and rising groups having increased risk compared with the falling group, with HR of 2.0 (CI: 1.1-3.1) and 23.0 (CI: 7.5-70.9), respectively. In the small number of patients in this study who had an extrapleural 


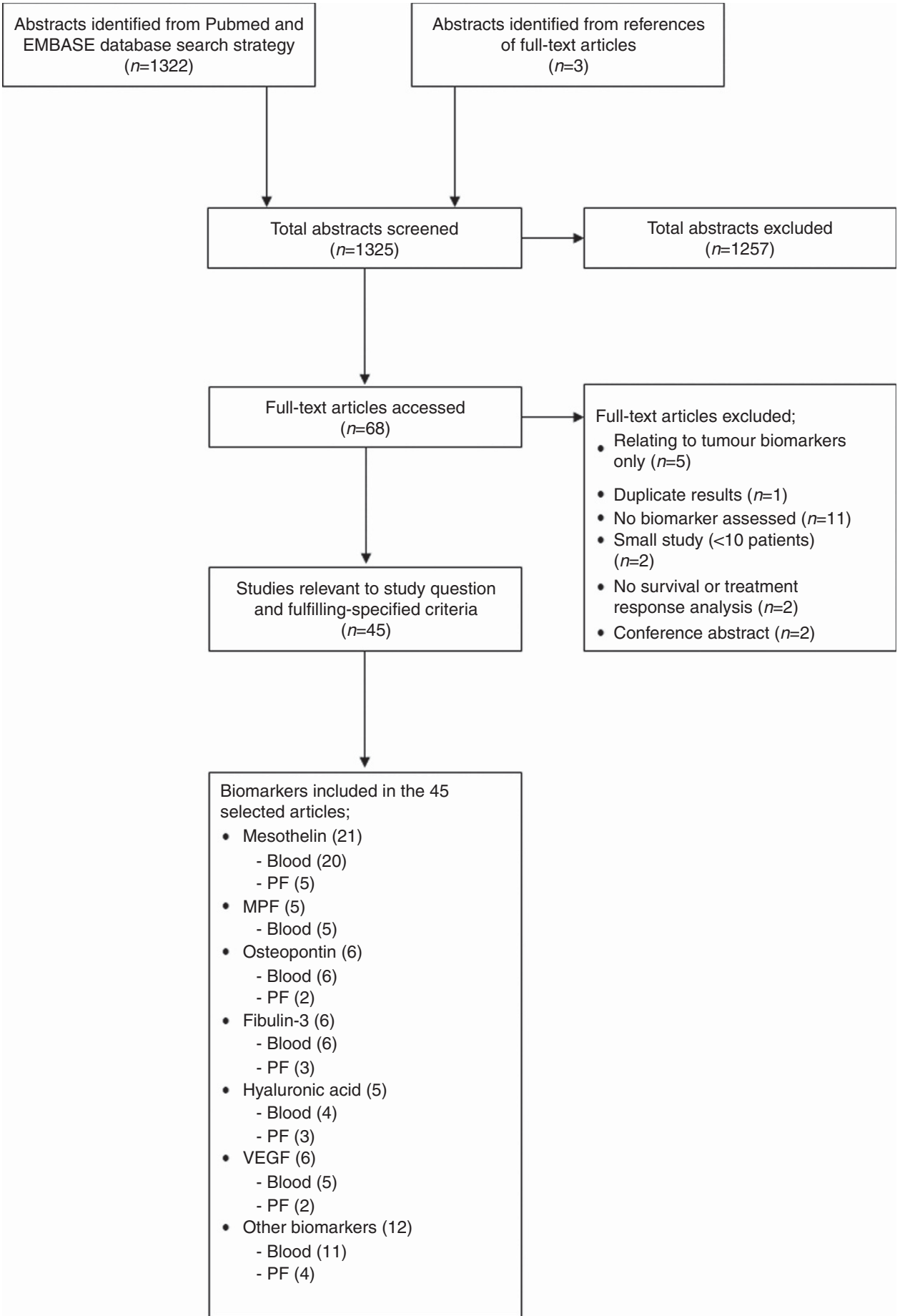

Figure 1. PRISMA flowchart.

pneumonectomy $(n=6)$, there was a mean $54 \%$ decrease in SM level pre to post surgery. There was no further testing of SM on these surgical patients to assess its role in predicting recurrent disease. Wheatley-Price et al (2010) published a similar study of 41 patients with non-sarcomatoid mesothelioma (39 pleural and 2 peritoneal) of whom $92 \%$ had an elevated baseline serum SM. Changes in SM or OPN were correlated with radiological reports (using descriptive reporting, RECIST and mod RECIST) during treatment. There was a significant association between relative and absolute change in SM and radiology reporting (all methods), the former having consistently better predictive value. Despite small numbers $(n=13)$, the same effect was seen in the BSC group with an average rise of SM by 26 and $60 \%$ for stable and progressed disease on mod RECIST criteria $(P=0.004)$. In total, this review identified eight studies that assessed the utility of serial serum SM testing in MPM. All found a correlation between falling SM levels and radiological response and/or improved OS (see Table 2).

Five studies (four prospective, one retrospective), with a total of 371 patients, investigated pf SM's ability to prognosticate from baseline (Creaney et al, 2007; Grigoriu et al, 2007; Yamada et al, 2011; Creaney et al, 2013, 2014). One study by Yamada et al (2011) found patients $(n=45)$ with higher pf SM levels survived significantly longer (dichotomy at $10 \mathrm{nM}$ ) at univariate but not 
Table 1. Full table of selected studies

\begin{tabular}{|c|c|c|c|c|c|}
\hline Author (year) & Study type & Biomarker & No. pts & Histology & Treatment \\
\hline Pass et al (2016) & Prospective & $\begin{array}{l}\text { SM (p) } \\
\text { Fibulin-3 (p) } \\
\text { OPN (p) }\end{array}$ & 194 & $\begin{array}{l}E-135 \\
N E-59\end{array}$ & $\begin{array}{c}\text { Surg }-100 \\
\text { C }-141 \\
\text { RTX }-68\end{array}$ \\
\hline Kaya et al (2015) & Prospective & Fibulin-3 (s) & 43 & $\begin{array}{c}E-30 \\
N E-13\end{array}$ & $\begin{array}{c}C-15 \\
\text { Surg }-7 \\
\text { BSC }-21\end{array}$ \\
\hline Fujimoto et al (2014) & Retrospective & $\begin{array}{l}\text { CD26 (s) } \\
\text { CD26 (pf) }\end{array}$ & 80 & $\begin{array}{c}E-53 \\
S-9 \\
B-18\end{array}$ & N/D \\
\hline Mundt et al (2014a) & Prospective & $\begin{array}{l}\text { Syndecan } 1 \text { (s) } \\
\text { OPN (s) } \\
\text { Syndecan } 1 \text { (pf) } \\
\text { OPN (pf) }\end{array}$ & $\begin{array}{l}91 \\
89\end{array}$ & $\begin{array}{l}E-62 \\
S-10 \\
B-13 \\
U n-95\end{array}$ & N/D \\
\hline Mundt et al (2014b) & Prespective & Novel proteomes (s) & 37 & $N / D$ & N/D \\
\hline Hassan et al (2014) & Prospective & $\begin{array}{l}\mathrm{SM}(\mathrm{s}) \\
\mathrm{MPF}(\mathrm{s}) \\
\mathrm{Ca125}(\mathrm{s})\end{array}$ & 24 & $N / D$ & $\mathrm{C} / \mathrm{Im}-24$ \\
\hline Creaney et al (2014) & Prospective & $\begin{array}{l}\text { Fibulin-3 (p) } \\
\text { SM (p) } \\
\text { Fibulin-3 (pf) } \\
\text { SM (pf) }\end{array}$ & 82 & $\begin{array}{c}E-32 \\
S-8 \\
B-13 \\
U-29\end{array}$ & $\begin{array}{c}C-37 \\
\text { Surg }-4 \\
\text { BSC }-37 \\
U-4\end{array}$ \\
\hline Creaney et al (2013) & Retrospective & $\begin{array}{l}\text { SM (s) } \\
\text { HA (s) } \\
\text { SM (pf) } \\
\text { HA (pf) }\end{array}$ & 96 & $\begin{array}{c}E-53 \\
S-2 \\
B-9 \\
U-32\end{array}$ & $N / D$ \\
\hline Tabata et al (2013) & Prospective & HMGB 1 (s) & 61 & $\begin{array}{c}E-43 \\
S-8 \\
B-6 \\
D-3 \\
A-1\end{array}$ & N/D \\
\hline Nowak et al (2013) & Prospective & $\begin{array}{l}\text { SM (s) } \\
\text { VEGF isoforms (s) } \\
\text { Interleukin-8 (s) } \\
\text { S-Kit (s) }\end{array}$ & 53 & $\begin{array}{c}E-39 \\
S-1 \\
B-10 \\
U n-3\end{array}$ & $\begin{array}{c}\text { Bio }-53 \\
\text { (second line) }\end{array}$ \\
\hline Franko et al (2012) & Prospective & $\mathrm{SM}(\mathrm{s})$ & 78 & $\begin{array}{l}E-64 \\
S-7 \\
B-7\end{array}$ & $\begin{array}{c}C-64 \\
\text { Surg }-10 \\
\text { BSC }-4\end{array}$ \\
\hline Kao et al (2012) & Prospective & $\begin{array}{l}\text { SM (s) } \\
\text { CRP (s) } \\
\text { IL-6 (s) } \\
\text { sIL-6r (s) } \\
\text { VEGF (s) }\end{array}$ & 63 & $\begin{array}{c}E-28 \\
S-4 \\
B-30\end{array}$ & $C-63$ \\
\hline Pass et al (2012) & Prospective & $\begin{array}{l}\text { Fibulin-3 (p) } \\
\text { Fibulin-3 (pf) }\end{array}$ & $\begin{array}{l}92 \\
74\end{array}$ & $N / D$ & N/D \\
\hline
\end{tabular}




\section{Table 1. (Continued)}

\begin{tabular}{|c|c|c|c|c|c|}
\hline Author (year) & Study type & Biomarker & No. pts & Histology & Treatment \\
\hline Ghanim et al (2012) & Retrospective & CRP (s) & 115 & $\begin{array}{l}E-80 \\
S-27 \\
U-8\end{array}$ & $\begin{array}{c}C+/- \text { RTX }-64 \\
\text { Surg }-51\end{array}$ \\
\hline Yamada et al (2011) & Prospective & SM (pf) & 45 & $\begin{array}{c}E-37 \\
S-5 \\
B-3\end{array}$ & N/D \\
\hline Hirayama et al (2011) & Prospective & VEGF (pf) & 46 & $\begin{array}{c}E-34 \\
S-10 \\
B-2\end{array}$ & N/D \\
\hline Hollevoet et al (2011) & Prospective & $\begin{array}{l}\mathrm{SM}(\mathrm{s}) \\
\operatorname{MPF}(\mathrm{s}) \\
\operatorname{OPN}(\mathrm{p})\end{array}$ & 62 & $\begin{array}{c}E-59 \\
S-1 \\
B-2\end{array}$ & $\begin{array}{c}C-48 \\
\text { Surg }-14\end{array}$ \\
\hline Creaney et al (2011) & Prospective & $\mathrm{SM}(\mathrm{s})$ & 95 & $\begin{array}{c}E-68 \\
S-9 \\
B-18\end{array}$ & $\begin{array}{c}C-61 \\
\text { Surg }-7 \\
\text { RTX }-2 \\
\text { BSC }-25\end{array}$ \\
\hline Grigoriu et al (2009) & Retrospective & $\mathrm{SM}(\mathrm{s})$ & 40 & $\begin{array}{l}E-35 \\
S-3 \\
B-2\end{array}$ & $\begin{array}{c}\mathrm{Im}-16 \\
\mathrm{C}-20 \\
\mathrm{BSC}-4\end{array}$ \\
\hline $\begin{array}{l}\text { Wheatley-Price et al } \\
(2010)\end{array}$ & Prospective & $\begin{array}{l}\text { SM (p) } \\
\text { OPN (p) }\end{array}$ & 41 & $E-41$ & $\begin{array}{c}C-21 \\
\text { Surg }-7 \\
\text { BSC - } 13\end{array}$ \\
\hline Schneider et al (2008) & Prospective & SM (s) & 129 & $\begin{array}{l}E-81 \\
S-14 \\
B-17 \\
U-17\end{array}$ & $\begin{array}{c}C-68 \\
\text { Surg }-41 \\
\text { RTX }-4 \\
\text { BSC - } 9 \\
\text { Un }-7\end{array}$ \\
\hline Grigoriu et al (2007) & Prospective & $\begin{array}{l}\text { SM (s) } \\
\text { OPN (s \& p) } \\
\text { SM (pf) } \\
\text { OPN (pf) }\end{array}$ & 96 & $\begin{array}{l}E-73 \\
S-10 \\
B-13\end{array}$ & $\begin{array}{c}C-70 \\
\text { Surg - } 10 \\
\text { BSC - } 16\end{array}$ \\
\hline Cristaudo et al (2007) & Prospective & SM (s) & 107 & $\begin{array}{c}E-72 \\
S-10 \\
B-7 \\
D-3 \\
U-15\end{array}$ & N/D \\
\hline Creaney et al (2007) & Prospective & $\begin{array}{l}\text { SM (s) } \\
\text { SM (pf) }\end{array}$ & 52 & $\begin{array}{c}E-15 \\
S-9 \\
B-5 \\
U-23\end{array}$ & N/D \\
\hline Filiberti et al (2005) & Prospective & PDGF-AB (s) & 93 & N/D & $\mathrm{N} / \mathrm{D}$ \\
\hline Robinson et al (2003) & Prospective & SM (s) & 44 & $\begin{array}{c}E-25 \\
S-4 \\
U-15\end{array}$ & $N / D$ \\
\hline Hedman et al (2003) & Retrospective & $\begin{array}{l}\mathrm{HA}(\mathrm{s}) \\
\text { Ca125 (s) } \\
\text { TPA (s) }\end{array}$ & 11 & $\mathrm{~N} / \mathrm{D}$ & N/D \\
\hline Strizzi et al (2001) & Retrospective & $\begin{array}{l}\text { VEGF (s) } \\
\text { VEGF (pf) }\end{array}$ & 12 & $\begin{array}{l}E-8 \\
S-1 \\
B-3\end{array}$ & N/D \\
\hline
\end{tabular}




\section{Table 1. (Continued)}

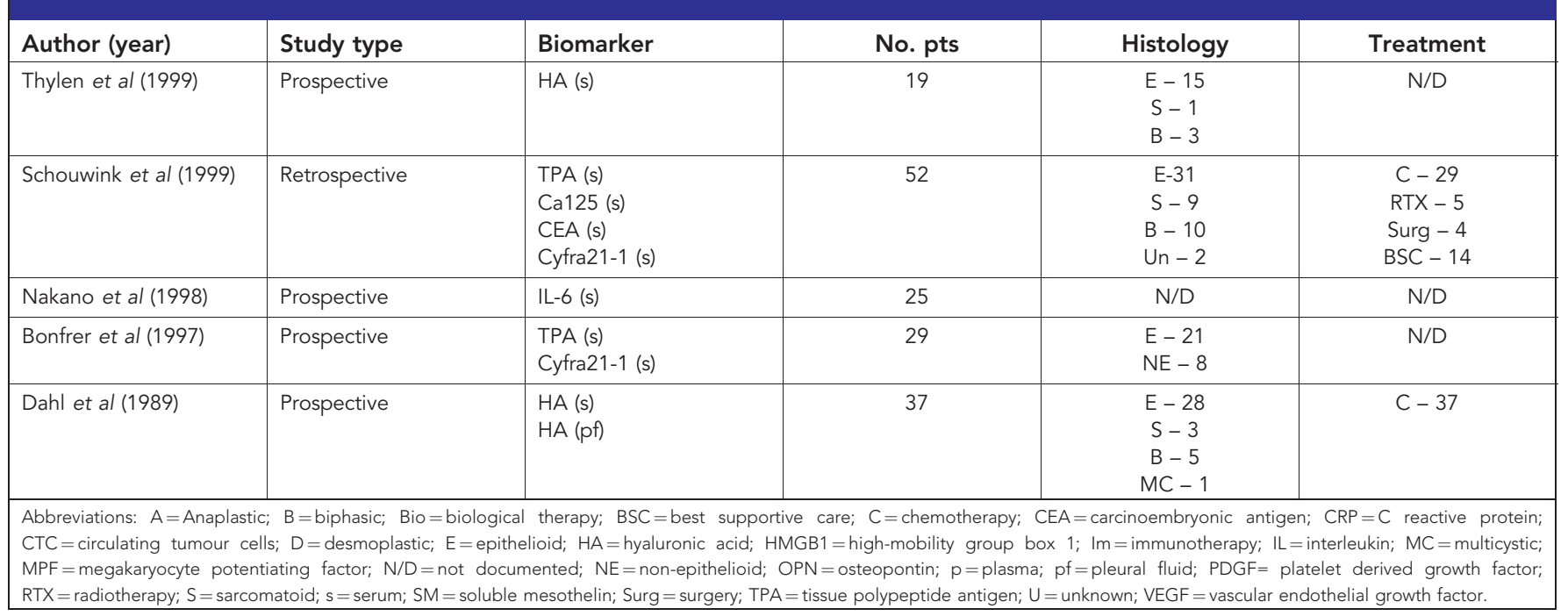

\section{Table 2. Studies assessing treatment response or survival using serial serum SM during treatment}

\begin{tabular}{|c|c|c|c|c|}
\hline Author (year) & Treatment (no. of patients) & Outcome measure & Threshold for SM change & Results \\
\hline Hooper et al (2015) & $P / C-58, B S C-15$ & $\begin{array}{l}\text { Mod RECIST CT } \\
\text { OS, TTP }\end{array}$ & $0 \%$ & $\begin{array}{l}\text { Chemotherapy group; a falling serum SM at } \\
6-8 \text { weeks was associated with longer time } \\
\text { to progression }(P<0.001) \text {, and a falling SM } \\
\text { post chemotherapy was associated with } \\
\text { improved OS }(P=0.031)\end{array}$ \\
\hline Hassan et al (2014) & $\mathrm{P} / \mathrm{C}$ and $\mathrm{Im}-20$ & Mod RECIST CT & $15 \%$ & $\begin{array}{l}\text { Fall in serum SM correlated with radiological } \\
\text { response with } 70 \% \text { accuracy }(P=0.003)\end{array}$ \\
\hline Franko et al (2012) & $\begin{array}{l}\mathrm{G} / \mathrm{C}-56, \mathrm{P} / \mathrm{C}-8, \mathrm{BSC}-4 \\
\text { Surg }-10\end{array}$ & Mod RECIST CT & $\mathrm{n} / \mathrm{a}$ & $\begin{array}{l}\text { Significantly lower mean serum SM in partial } \\
\text { response or stable disease compared to } \\
\text { progressive disease }(P=0.001)\end{array}$ \\
\hline Hollevoet et al (2011) & $P / C-57$, Surg -5 & Mod RECIST CT & $15 \%$ & $\begin{array}{l}\text { Partial response to chemotherapy } \\
\text { correlated with a } 34 \% \text { fall in SM }(P=0.010) \\
\text { compared with a } 54 \% \text { rise in progressive } \\
\text { disease }(P<0.001)\end{array}$ \\
\hline $\begin{array}{l}\text { Wheatley-Price et al } \\
\text { (2010) }\end{array}$ & Chemo - 21, BSC - 13, Surg - 8 & $\begin{array}{l}\text { Mod RECIST CT } \\
\text { RECIST CT } \\
\text { CT report }\end{array}$ & $10 \%$ or $5 \mathrm{nmoll} \mathrm{I}^{-1}$ & $\begin{array}{l}\text { Chemotherapy and BSC groups; relative } \\
\text { change in serum SM from baseline } \\
\text { significantly associated with disease } \\
\text { progression }(P<0.010)\end{array}$ \\
\hline Grigoriu et al (2009) & Chemo - 20, Im - 16, BSC - 4 & Mod RECIST CT & $10 \%$ & $\begin{array}{l}\text { In patients with raised SM at baseline } \\
\left(>1 \mathrm{nMI}^{-1}\right) \text {, rising level correlated with } \\
\text { progressive disease in } 12 \text { out of } 16 \text { patients. } \\
\text { OS higher in patients with stable SM } \\
\text { compared with increasing }(P=0.012)\end{array}$ \\
\hline
\end{tabular}

multivariate analysis. This finding was not replicated in the other larger studies, which found no relationship between pf SM and OS.

Megakaryocyte potentiating factor (MPF) or N-ERC/mesothelin. All five studies (five prospective), with a total of 232 patients, involving serum MPF were published after 2008 (Tajima et al,
2008; Hollevoet et al, 2011, 2012; Mori et al, 2013; Hassan et al, 2014). The earliest by Tajima et al (2008) included 14 patients with MPM receiving a variety of chemotherapeutic regimens and tested MPF and OPN prior to and following treatment. Despite small numbers, the ratio between levels before and after therapy was lower in those who had progressed on RECIST criteria (i.e., levels 
had risen) compared with those with a partial response $(P<0.05)$. A larger study was performed by Hollevoet et al (2011) on 62 patients receiving either extrapleural pneumonectomy $(n=14)$ or pemetrexed/platinum-based chemotherapy $(n=48)$. Patients had modified RECIST CTs and matched MPF, SM and OPN levels (no greater than 3 weeks apart) before and after treatment. In the surgical group, only five patients had pre- and post-treatment samples and median levels of both MPF and SM fell by $76 \%$ and $78 \%$, respectively (median OPN levels actually rose by $20 \%$ ). In the chemotherapy group, the authors classified a change in biomarker level as a change of $>15 \%$ from baseline. They demonstrated that serum MPF (and SM) could predict treatment response, with a median $53 \%$ fall in partial response $(n=14)$ compared with $58 \%$ rise in progressive disease $(n=16)$. This study did not show any correlation between baseline MPF and OS. However, a study from the same author investigated the effect of age, BMI and renal function on serum MPF and SM levels, finding that only renal function altered biomarker levels in 106 MPM patients (a worsening renal function increased serum MPF and SM) (Hollevoet et al, 2012). Once this and other covariates were considered, baseline serum MPF (and not SM) was found to correlate with OS $(P=0.040)$. Finally, in a Phase 1 dose escalation study of an anti-mesothelin immunotherapy called SS1P (in combination with Pemetrexed and Cisplatin), the serum biomarkers MPF, SM and Ca125 were tested for correlation with treatment response (mod RECIST CT) (Hassan et al, 2014). Twenty patients were evaluable with biomarkers pre and post treatment. All three biomarkers showed 'strong significant correlation' with partial response, stable disease or progressive disease. The biomarkers' accuracy in predicting response on CT was assessed with $15 \%$ used as a cut-off for change in biomarker levels from baseline. Megakaryocyte potentiating factor correctly classified 15 out of 20 patients (75\% accuracy) as having progressive or stable disease (with rising or stable/ falling levels, respectively) based on their CT scan, compared with 14 out of $20(70 \%)$ and 12 out of $20(60 \%)$ for SM and Ca125, respectively.

Osteopontin (OPN). All six of the studies (six prospective) that assessed serum/plasma OPN, with a total of 498 patients, were looking at its role alongside other biomarkers. Two of these studies, total of 185 patients, also examined pf OPN (Grigoriu et al, 2007; Tajima et al, 2008; Wheatley-Price et al, 2010; Hollevoet et al, 2011; Abakay et al, 2014; Mundt et al, 2014a; Pass et al, 2016). Grigoriu et al (2007) measured baseline serum and pf OPN and SM (SM results above) in a cohort of $96 \mathrm{MPM}$ patients. Baseline serum OPN had a statistically significant relationship with OS at multivariate analysis in a model that included tumour histology (HR: 3.46, CI: 1.1-10.9, $P=0.034$ ). Using a cut-off of $350 \mathrm{ng} \mathrm{ml}^{-1}$ (selected using a maximisation of HR model), patients with a lowserum OPN had a median OS of 15 months compared with 5 months in high-serum OPN levels. Pleural fluid OPN was also measured and was not found to correlate with OS. Serum OPN's ability to act as a baseline prognostic marker was replicated by Hollevoet et al (2011) (study discussed above). Baseline OPN correlated with both OS and PFS (optimum cut-off for their data set was $863 \mathrm{ng} \mathrm{ml}^{-1}$ ) and appeared to be an independent factor with no correlation with other biomarkers or tumour stage. More recently, Pass et al investigated the benefit of adding baseline plasma biomarker levels to previously validated prognostic tools (EORTC prognostic index of mesothelioma and the CALGB index). In a discovery cohort of 83 patients, of whom two-thirds had cytoreductive surgery, baseline levels of plasma OPN, SM and fibulin-3 were measured. Both the plasma OPN and SM, but not fibulin-3, were independently correlated with OS. Interestingly, in a prognostic model including well known poor prognostic indicators such as low $\mathrm{Hb}$ and EORTC score $(>1.27)$ only high
OPN remained an independently significant predictor of worse prognosis.

A study by Mundt et al (2014a) analysed serum and pf OPN from two separate cohorts to assess its role in diagnosis and prognosis at baseline. Although the diagnostic analysis of serum OPN involved 91 patients with MPM, full survival analysis was only available for 19 patients. Despite this data attrition, serum OPN was found to correlate with OS using a $185 \mathrm{ng} \mathrm{ml}^{-1}$ cut-off (HR: 2.5, CI: 1.4-10.3), the median of the data set. In the pf cohort, 40 patients had survival data available and using pf OPN cut-off of $1.6 \mu \mathrm{g} \mathrm{ml}^{-1}$ resulted in median OS times of $29 \mathrm{~m} v s 13 \mathrm{~m}$ for low to high levels, respectively (HR: 2.2, CI: 1.2-4.2). However, neither of these correlations were assessed using multivariate analysis or evaluated alongside tumour histology.

Fibulin-3. Six studies (five prospective, one retrospective) involving serum/plasma fibulin-3 were found by this systematic review, comprising 568 patients overall (Pass et al, 2012; Creaney et al, 2014; Hooper et al, 2015; Kaya et al, 2015; Kirschner et al, 2015; Pass et al, 2016). Of these, three studies (two prospective, one retrospective), with a total of 186 patients, also measured pf fibulin. No study found serum/plasma fibulin-3 to be a significant marker of prognostication at baseline. Hooper et al (2015) measured serum fibulin-3 before, during (after two cycles of Pem/Cis) and after chemotherapy. Baseline levels were higher in the epithelioid subtypes but there was no correlation with OS when histological subtypes were analysed separately. In addition, serial sampling did not predict treatment response or PFS.

The earliest study to analyse pf fibulin-3 as a prognostic marker was carried out by Pass et al (2012). The primary outcome was fibulin-3's diagnostic utility, so survival data was only available for a proportion of the pf cohort $(n=54)$. PF fibulin-3 correlated with pathological stage, with stages 1 and $2(n=21)$ having a median level of $576 \mathrm{ng} \mathrm{ml}^{-1}$ compared with $765 \mathrm{ng} \mathrm{ml}^{-1}$ in stages 3 and $4(P=0.040)$. When pf fibulin-3 levels were dichotomised at the database median of $733.4 \mathrm{ng} \mathrm{ml}^{-1}$ a low baseline level inferred better OS and this remained significant in a multivariate model that included gender, stage and histological subtype $(P=0.024)$. Creaney et al (2014) also measured both serum and pf fibulin-3 in a prospectively collected cohort of 82 patients with MPM with a focus on diagnostic utility but with follow-up data for the majority of the cohort $(n=78)$. Patients with biphasic or sarcomatoid histology had significantly $(P=0.002)$ higher pf fibulin-3 concentrations (median $1331 \mathrm{ng} \mathrm{ml}^{-1}$ ) compared with epithelioid subtypes (median $426 \mathrm{ng} \mathrm{ml}^{-1}$ ), but no relationship to tumour stage. A linear negative relation was found between OS and pf fibulin-3 and remained significant at multivariate analysis $(P=0.017)$. Lastly, a retrospective analysis of three cohorts of MPM patients (serum $n=37$ and $n=47$, pf $n=30$ ) found that lower pf fibulin-3, but not serum, was associated with improved OS at multivariate analysis (Kirschner et al, 2015).

Hyaluronic acid (HA). Four studies (two prospective, two retrospective), with a total of 163 patients, assessed the utility of serum HA between 1989 and 2013 (Dahl et al, 1989; Thylen et al, 1999; Hedman et al, 2003; Creaney et al, 2013). Pleural fluid HA was measured in three of the selected studies (one prospective, two retrospective), with a total of 233 patients (Dahl et al, 1989; Thylen et al, 2001; Creaney et al, 2013). The earliest study, from Dahl et al (1989), measured serial serum HA in patients undergoing methotrexate therapy for pleural $(n=34)$ or peritoneal $(n=3)$ mesothelioma. They showed that serum HA were higher in later disease stage but presented no data regarding histological subtypes. Additionally, in patients who were deemed to have progressed, based on subjective CT reporting $(n=13)$, levels of HA rose (median $=25$, IQR 6-37) compared with falling levels in responders $(n=20)$ (median $=-5$, IQR: $-14-3$ ). Pleural fluid HA did not correlate with serum levels and there was no relationship between tumour stage or disease response. In contrast, 
a more recent case series retrospectively analysed serum and pf HA in 96 MPM cases (Creaney et al, 2013). In this study, serum HA was not significantly raised in MPM patients compared with patients with benign conditions or lung cancer with no relationship with OS. Pleural fluid HA was significantly higher in MPM patients and demonstrated a biphasic distribution that was independent of tumour histology. Although no treatment data was presented, using a cut-off of $75 \mathrm{mg} \mathrm{ml}^{-1}$, there was a survival benefit for high pf HA levels (18 months) compared with low levels (12.6 months). The phenomena of high pf HA and improved survival is replicated by Thylen et al (2001) who measured pf levels in patients receiving either chemotherapy $(n=56)$ or BSC $(n=44)$, although histological subtypes were not analysed separately.

Vascular endothelial growth factor (VEGF). This systematic review identified six papers involving serum/plasma (four prospective, one retrospective) or pf (one retrospective, one prospective) vascular endothelial growth factor (VEGF) levels, with a total of 287 and 53 patients, respectively, (Strizzi et al, 2001; Yasumitsu et al, 2010; Hirayama et al, 2011; Kao et al, 2012; Kindler et al, 2012; Nowak et al, 2013). A 7-year prospective single centre case series of 51 patients with MPM analysed serum pan-VEGF levels and compared them to a non-MPM asbestosexposed population $(n=42)$ (Yasumitsu et al, 2010). Serum VEGF levels were significantly higher in the MPM population and increased with tumour stage. Median levels were higher in epithelioid $v s$ sarcomatoid histology $\left(1071 \mathrm{pg} \mathrm{ml}^{-1}\right.$ vs $580 \mathrm{pg} \mathrm{ml}^{-1}$, respectively) but because of low numbers of sarcomatoid cases $(n=6)$, this result was not statistically significant. At multivariate analysis, there was no significant correlation with OS. Kao et al (2012) analysed a variety of novel biomarkers (pan-VEGF, CRP, IL-6, sIL-6R and SM) in a nonrandomised trial of thalidomide as a chemotherapy adjunct $(n=34)$ or single agent $(n=29)$. At multivariate analysis, baseline serum VEGF was the only significant biomarker in predicting OS $(P=0.025)$, with higher median survival in lower VEGF levels. In addition, VEGF levels were tested post chemotherapy (at 8 weeks). Patients with high baseline levels that subsequently fell had median OS of 79 weeks compared with 39 weeks $(P=0.050)$.

A phase II trial of second-line therapy (Sunitinib Malate, a multitargeted tyrosine kinase inhibitor) robustly assessed the role of several serum VEGF isoforms in prognostication (Nowak et al, 2013). Fifty-three patients with progression following conventional chemotherapy were enroled, with only one patient with sarcomatoid MPM meeting eligibility criteria. Several serum VEGF isoforms were tested (A, C, R2 and R3) as well as SM, c-kit and IL-8 at baseline, 6 weeks and every 12 weeks thereafter. Baseline VEGF-A and VEGF-R2 were predictive of radiological response at multivariate analysis, with only percentage change in SM being associated with time to progression (HR: 3.84, $P<0.001$ ). Another trial of biological therapy compared bevacizumab (a monoclonal antibody to VEGF) to placebo when added to chemotherapy with gemcitabine and cisplatin in 108 patients with MPM (Kindler et al, 2012). Vascular endothelial growth factor levels were measured pre-treatment in 56 patients. The trial found no difference between the two treatment arms with partial response rates of $24.5 \%$ and $21.8 \%$ for the bevacizumab and placebo arms, respectively, $(P=0.74)$. There was no significant difference in baseline VEGF levels between responders and non-responders, although higher baseline levels were associated with worse PFS $(P=0.049)$ and OS $(P=0.014)$.

A diagnostic study measured pf pan-VEGF levels in 46 MPM patients (Hirayama et al, 2011). In the 28 patients followed up to 600 days, those with a pf VEGF $>2000 \mathrm{pg} \mathrm{ml}^{-1}$ (a pre-defined cut-off) had lower OS at multivariate analysis (HR; 961.2, CI: 7.1$130446, P=0.006)$.

\section{DISCUSSION}

This systematic review identified 45 studies from the current literature that assessed the prognostic or treatment monitoring ability of biomarkers in MPM. There was significant variation in the quality of the selected studies with many having a moderate to high risk of bias due to study attrition or lack of reporting of confounding factors (as evidenced by the variation in QUIPS scores). In addition, there was considerable heterogeneity within studies regarding patient treatment (variation in numbers undergoing standard chemotherapy, trial drugs, surgery or BSC), which is a major confounder in prognostic studies that can only be partly adjusted for using multivariate analysis. Several papers combined these groups when assessing a biomarker's baseline prognostic ability, making many of their conclusions invalid.

Robust methodology was used to capture all available literature, including an evidence-based search strategy, multiple independent reviewers, PRISMA reporting methodology and the use of the QUIPS tool for study assessment. However, given the inter-study variability in biomarker cut-offs, histological subtypes, treatment modalities and outcome measures (OS, PFS or radiological treatment response) no attempt to meta-analyse the studies was made.

The majority of selected studies examined the utility of serum SM as a baseline prognostic indictor, often as a secondary outcome to its diagnostic utility. Earlier case series suggested higher baseline levels inferred a worse prognosis, but this finding was inconsistently replicated by more recent studies. Soluble mesothelin is only expressed by tumours with full or partial epithelioid histology, so variation between studies in histological subtypes has a significant impact on the interpretation of these results. Several studies have demonstrated no correlation with OS when tumour histology and renal function (renal function is inversely correlated with serum $\mathrm{SM}$ ) are included in multivariate analyses. When serum SM is measured serially it has been consistently shown to correlate with changes in modified RECIST CT findings or survival (OS and PFS). The eight studies examining this relationship focused on patients receiving chemotherapy, with only 34 patients having had surgery. Between studies, there was variation in the thresholds used to define a significant change in serum SM as well as the appropriate sampling intervals during or after treatment. Before serial SM testing can be recommended in routine clinical practice a large prospective study is required to address these uncertainties and assess its use in surgical and BSC cohorts. Megakaryocyte potentiating factor is formed from the same precursor protein as SM and is a more novel biomarker in MPM (Hollevoet et al, 2010). Megakaryocyte potentiating factor and SM correlated strongly in the three studies that measured both concurrently but the strength of evidence for serum MPF is far smaller than for SM.

This systematic review identified several studies that correlated high OPN levels with poor prognosis, including within prognostic tools. Also plasma OPN showed no correlation with other biomarkers, indicating that it may offer independent prognostic information. There was significant variability in the cut-offs used between studies, which is likely reflective of the variation in treatment modality between cohorts as well as the ELISA platforms used (Anborgh et al, 2009). In addition, because OPN is cleaved by thrombin following blood coagulation, plasma sampling is superior to serum (Pass et al, 2016). The majority of studies found by this review analysed plasma OPN, but in order for this biomarker to be validated in the future a consensus approach is required for sampling and analysis.

Serum fibulin-3 has shown promise as a diagnostic biomarker (Pass et al, 2012) but was not a marker of prognosis on the basis of this systematic review. However, higher levels of fibulin-3 in pf did inversely correlate with survival, although this is likely in part due 
to the much higher levels found in effusions of the more aggressive sarcomatoid MPM.

Vascular endothelial growth factor is a well-documented marker of tumour angiogenesis and is raised in the serum of patients with MPM (Strizzi et al, 2001). It is of particular importance in MPM given the emergence of antiangiogenic VEGF-targeted treatments that have been shown to improve survival when given in combination with pemetrexed and cisplatin (Zalcman et al, 2016). The studies involving serum VEGF were heterogeneous in terms of design but showed positive results for pan-VEGF and its isoforms as prognostic or monitoring biomarkers. No studies demonstrated any ability of serum VEGF to select responders from non-responders for biologic therapy, but this area demands further study given the development of promising but expensive biologicals (Kindler et al, 2012; Nowak et al, 2013).

In conclusion, from the 45 studies published in the literature no serum or pf biomarker was identified that could be recommended currently for use in clinical practice. There was considerable heterogeneity within studies for patient treatment, tumour histology and follow-up, as well as inter-study variability in terms of biomarker cut-offs. Serum SM when measured before and after treatment has been shown to track treatment response but further studies are required to ascertain its place in the chemotherapy or surgical management pathway. Serum OPN showed an ability to prognosticate from baseline, but whether this has clinical utility is uncertain. With considerable variation in response rates to chemotherapy and the emergence of promising biological therapies, biomarkers that could select responders from non-responders at baseline or during treatment would aid clinical decision making, prevent patients getting ineffective therapy and improve cost effectiveness.

\section{ACKNOWLEDGEMENTS}

DTA is funded by a National Institute for Health Research (NIHR) Academic Clinical Fellowship. NMR is funded by the Oxford NIHR Biomedical Research Centre.

\section{CONFLICT OF INTEREST}

The authors declare no conflict of interest.

\section{DISCLAIMER}

This article presents independent research funded by the National Institute for Health Research (NIHR). The views expressed are those of the author(s) and not necessarily those of the NHS, the NIHR or the Department of Health.

\section{REFERENCES}

Abakay O, Tanrikulu AC, Palanci Y, Abakay A (2014) The value of inflammatory parameters in the prognosis of malignant mesothelioma. J Int Med Res 42(2): 554-565.

Altman DG (2001) Systematic reviews of evaluations of prognostic variables. BMJ 323(7306): 224-228.

Anborgh PH, Wilson SM, Tuck AB, Winquist E, Schmidt N, Hart R, Kon S, Maeda M, Uede T, Stitt LW, Chambers AF (2009) New dual monoclonal ELISA for measuring plasma osteopontin as a biomarker associated with survival in prostate cancer: clinical validation and comparison of multiple ELISAs. Clin Chem 55(5): 895-903.

Armato 3rd SG, Ogarek JL, Starkey A, Vogelzang NJ, Kindler HL, Kocherginsky M, MacMahon H (2006) Variability in mesothelioma tumor response classification. AJR Am J Roentgenol 186(4): 1000-1006.
Arnold DT, Hooper CE, Morley A, White P, Lyburn ID, Searle J, Darby M, Hall T, Hall D, Rahman NM, De Winton E, Clive A, Masani V, Dangoor A, Guglani S, Jankowska P, Lowndes SA, Harvey JE, Braybrooke JP, Maskell NA (2015) The effect of chemotherapy on health-related quality of life in mesothelioma: results from the SWAMP trial. Br J Cancer 112(7): 1183-1189.

Bonfrer JM, Schouwink JH, Korse CM, Baas P (1997) Cyfra 21-1 and TPA as markers in malignant mesothelioma. Anticancer Res 17(4B): 2971-2973.

Chapman A, Mulrennan S, Ladd B, Muers MF (2008) Population based epidemiology and prognosis of mesothelioma in Leeds, UK. Thorax 63(5): 435-439.

Creaney J, Dick IM, Meniawy TM, Leong SL, Leon JS, Demelker Y, Segal A, Musk AW, Lee YC, Skates SJ, Nowak AK, Robinson BW (2014) Comparison of fibulin-3 and mesothelin as markers in malignant mesothelioma. Thorax 69(10): 895-902.

Creaney J, Dick IM, Segal A, Musk AW, Robinson BW (2013) Pleural effusion hyaluronic acid as a prognostic marker in pleural malignant mesothelioma. Lung Cancer 82(3): 491-498.

Creaney J, Francis RJ, Dick IM, Musk AW, Robinson BW, Byrne MJ, Nowak AK (2011) Serum soluble mesothelin concentrations in malignant pleural mesothelioma: relationship to tumor volume, clinical stage and changes in tumor burden. Clin Cancer Res 17(5): 1181-1189.

Creaney J, Yeoman D, Naumoff LK, Hof M, Segal A, Musk AW, De Klerk N, Horick N, Skates SJ, Robinson BW (2007) Soluble mesothelin in effusions: a useful tool for the diagnosis of malignant mesothelioma. Thorax 62(7): 569-576.

Cristaudo A, Foddis R, Vivaldi A, Guglielmi G, Dipalma N, Filiberti R, Neri M, Ceppi M, Paganuzzi M, Ivaldi GP, Mencoboni M, Canessa PA, Ambrosino N, Chella A, Mutti L, Puntoni R (2007) Clinical significance of serum mesothelin in patients with mesothelioma and lung cancer. Clin Cancer Res 13(17): 5076-5081.

Dahl IM, Solheim OP, Erikstein B, Muller E (1989) A longitudinal study of the hyaluronan level in the serum of patients with malignant mesothelioma under treatment. Hyaluronan as an indicator of progressive disease. Cancer 64(1): 68-73.

Dretzke J, Ensor J, Bayliss S, Hodgkinson J, Lordkipanidze M, Riley RD, Fitzmaurice D, Moore D (2014) Methodological issues and recommendations for systematic reviews of prognostic studies: an example from cardiovascular disease. Syst Rev 3: 140.

Filiberti R, Marroni P, Neri M, Ardizzoni A, Betta PG, Cafferata MA, Canessa PA, Puntoni R, Ivaldi GP, Paganuzzi M (2005) Serum PDGF-AB in pleural mesothelioma. Tumour Biol 26(5): 221-226.

Franko A, Dolzan V, Kovac V, Arneric N, Dodic-Fikfak M (2012) Soluble mesothelin-related peptides levels in patients with malignant mesothelioma. Dis Markers 32(2): 123-131.

Fujimoto N, Ohnuma K, Aoe K, Hosono O, Yamada T, Kishimoto T, Morimoto C (2014) Clinical significance of soluble CD26 in malignant pleural mesothelioma. PLoS ONE 9(12): e115647.

Ghanim B, Hoda MA, Klikovits T, Winter MP, Alimohammadi A, Grusch M, Dome B, Arns M, Schenk P, Jakopovic M, Samarzija M, Brcic L, Filipits M, Laszlo V, Klepetko W, Berger W, Hegedus B (2014) Circulating fibrinogen is a prognostic and predictive biomarker in malignant pleural mesothelioma. Br J Cancer 110(4): 984-990.

Ghanim B, Hoda MA, Winter MP, Klikovits T, Alimohammadi A, Hegedus B, Dome B, Grusch M, Arns M, Schenk P, Pohl W, Zielinski C, Filipits M, Klepetko W, Berger W (2012) Pretreatment serum C-reactive protein levels predict benefit from multimodality treatment including radical surgery in malignant pleural mesothelioma: a retrospective multicenter analysis. Ann Surg 256(2): 357-362.

Grigoriu BD, Chahine B, Vachani A, Gey T, Conti M, Sterman DH, Marchandise G, Porte H, Albelda SM, Scherpereel A (2009) Kinetics of soluble mesothelin in patients with malignant pleural mesothelioma during treatment. Am J Respir Crit Care Med 179(10): 950-954.

Grigoriu BD, Scherpereel A, Devos P, Chahine B, Letourneux M, Lebailly P, Gregoire M, Porte H, Copin MC, Lassalle P (2007) Utility of osteopontin and serum mesothelin in malignant pleural mesothelioma diagnosis and prognosis assessment. Clin Cancer Res 13(10): 2928-2935.

Hassan R, Sharon E, Thomas A, Zhang J, Ling A, Miettinen M, Kreitman RJ Steinberg SM, Hollevoet K, Pastan I (2014) Phase 1 study of the antimesothelin immunotoxin SS1P in combination with pemetrexed and cisplatin for front-line therapy of pleural mesothelioma and correlation of tumor response with serum mesothelin, megakaryocyte potentiating factor, and cancer antigen 125. Cancer 120(21): 3311-3319. 
Hayden JA, van der Windt DA, Cartwright JL, Cote P, Bombardier C (2013) Assessing bias in studies of prognostic factors. Ann Intern Med 158(4): 280-286.

Hedman M, Arnberg H, Wernlund J, Riska H, Brodin O (2003) Tissue polypeptide antigen (TPA), hyaluronan and CA 125 as serum markers in malignant mesothelioma. Anticancer Res 23(1B): 531-536.

Hirayama N, Tabata C, Tabata R, Maeda R, Yasumitsu A, Yamada S, Kuribayashi K, Fukuoka K, Nakano T (2011) Pleural effusion VEGF levels as a prognostic factor of malignant pleural mesothelioma. Respir Med 105(1): 137-142.

Hollevoet K, Nackaerts K, Gosselin R, De Wever W, Bosquee L, De Vuyst P, Germonpre P, Kellen E, Legrand C, Kishi Y, Delanghe JR, van Meerbeeck JP (2011) Soluble mesothelin, megakaryocyte potentiating factor, and osteopontin as markers of patient response and outcome in mesothelioma. J Thorac Oncol 6(11): 1930-1937.

Hollevoet K, Nackaerts K, Thas O, Thimpont J, Germonpre P, De Vuyst P, Bosquee L, Legrand C, Kellen E, Kishi Y, Delanghe JR, van Meerbeeck JP (2012) The effect of clinical covariates on the diagnostic and prognostic value of soluble mesothelin and megakaryocyte potentiating factor. Chest 141(2): 477-484.

Hollevoet K, Nackaerts K, Thimpont J, Germonpre P, Bosquee L, De Vuyst P, Legrand C, Kellen E, Kishi Y, Delanghe JR, van Meerbeeck JP (2010) Diagnostic performance of soluble mesothelin and megakaryocyte potentiating factor in mesothelioma. Am J Respir Crit Care Med 181(6): 620-625.

Hooper CE, Lyburn ID, Searle J, Darby M, Hall T, Hall D, Morley A, White P, Rahman NM, De Winton E, Clive A, Masani V, Arnold DT, Dangoor A, Guglani S, Jankowska P, Lowndes SA, Harvey JE, Braybrooke JP, Maskell NA (2015) The south west area mesothelioma and pemetrexed trial: a multicentre prospective observational study evaluating novel markers of chemotherapy response and prognostication. Br J Cancer 112(7): 11751182 .

Kao SC, Harvie R, Paturi F, Taylor R, Davey R, Abraham R, Clarke S, Marx G, Cullen M, Kerestes Z, Pavlakis N (2012) The predictive role of serum VEGF in an advanced malignant mesothelioma patient cohort treated with thalidomide alone or combined with cisplatin/gemcitabine. Lung Cancer 75(2): 248-254.

Kaya H, Demir M, Taylan M, Sezgi C, Tanrikulu AC, Yilmaz S, Bayram M, Kaplan I, Senyigit A (2015) Fibulin-3 as a diagnostic biomarker in patients with malignant mesothelioma. Asian Pac J Cancer Prev 16(4): 1403-1407.

Kindler HL, Karrison TG, Gandara DR, Lu C, Krug LM, Stevenson JP, Janne PA, Quinn DI, Koczywas MN, Brahmer JR, Albain KS, Taber DA, Armato 3rd SG, Vogelzang NJ, Chen HX, Stadler WM, Vokes EE (2012) Multicenter, double-blind, placebo-controlled, randomized phase II trial of gemcitabine/cisplatin plus bevacizumab or placebo in patients with malignant mesothelioma. J Clin Oncol 30(20): 2509-2515.

Kirschner MB, Pulford E, Hoda MA, Rozsas A, Griggs K, Cheng YY, Edelman JJ, Kao SC, Hyland R, Dong Y, Laszlo V, Klikovits T, Vallely MP, Grusch M, Hegedus B, Dome B, Klepetko W, van Zandwijk N, Klebe S, Reid G (2015) Fibulin-3 levels in malignant pleural mesothelioma are associated with prognosis but not diagnosis. Br J Cancer 113(6): 963-969.

Linch M, Gennatas S, Kazikin S, Iqbal J, Gunapala R, Priest K, Severn J, Norton A, Ayite B, Bhosle J, O’Brien M, Popat S (2014) A serum mesothelin level is a prognostic indicator for patients with malignant mesothelioma in routine clinical practice. BMC Cancer 14: 674 .

Mori T, Tajima K, Hirama M, Sato T, Kido K, Iwakami S, Sasaki S, Iwase A, Shiomi K, Maeda M, Hino O, Takahashi K (2013) The N-ERC index is a novel monitoring and prognostic marker for advanced malignant pleural mesothelioma. J Thorac Dis 5(2): 145-148.

Mundt F, Heidari-Hamedani G, Nilsonne G, Metintas M, Hjerpe A, Dobra K (2014a) Diagnostic and prognostic value of soluble syndecan-1 in pleural malignancies. Biomed Res Int 2014: 419853.

Mundt F, Johansson HJ, Forshed J, Arslan S, Metintas M, Dobra K, Lehtio J, Hjerpe A (2014b) Proteome screening of pleural effusions identifies galectin 1 as a diagnostic biomarker and highlights several prognostic biomarkers for malignant mesothelioma. Mol Cell Proteomics 13(3): 701-715.

Nakano T, Chahinian AP, Shinjo M, Tonomura A, Miyake M, Togawa N, Ninomiya K, Higashino K (1998) Interleukin 6 and its relationship to clinical parameters in patients with malignant pleural mesothelioma. $\mathrm{Br} J$ Cancer 77(6): 907-912.

Nowak AK, Brown C, Millward MJ, Creaney J, Byrne MJ, Hughes B, Kremmidiotis G, Bibby DC, Leske AF, Mitchell PL, Pavlakis N, Boyer M,
Stockler MR (2013) A phase II clinical trial of the vascular disrupting agent BNC105P as second line chemotherapy for advanced malignant pleural mesothelioma. Lung Cancer 81(3): 422-427.

Pass HI, Goparaju C, Espin-Garcia O, Donington J, Carbone M, Patel D, Chen Z, Feld R, Cho J, Gadgeel S, Wozniak A, Chachoua A, Leighl N, Tsao MS, de Perrot M, Xu W, Liu G (2016) Plasma biomarker enrichment of clinical prognostic indices in malignant pleural mesothelioma. J Thorac Oncol 11(6): 900-909.

Pass HI, Levin SM, Harbut MR, Melamed J, Chiriboga L, Donington J, Huflejt M, Carbone M, Chia D, Goodglick L, Goodman GE, Thornquist MD, Liu G, de Perrot M, Tsao MS, Goparaju C (2012) Fibulin-3 as a blood and effusion biomarker for pleural mesothelioma. N Engl J Med 367(15): $1417-1427$

Pass HI, Lott D, Lonardo F, Harbut M, Liu Z, Tang N, Carbone M, Webb C, Wali A (2005) Asbestos exposure, pleural mesothelioma, and serum osteopontin levels. N Engl J Med 353(15): 1564-1573.

Pass HI, Wali A, Tang N, Ivanova A, Ivanov S, Harbut M, Carbone M, Allard J (2008) Soluble mesothelin-related peptide level elevation in mesothelioma serum and pleural effusions. Ann Thorac Surg 85(1): 265-272.

Raphael J, Massard C, Gong IY, Farace F, Margery J, Billiot F, Hollebecque A, Besse B, Soria JC, Planchard D (2015) Detection of circulating tumour cells in peripheral blood of patients with malignant pleural mesothelioma. Cancer Biomark 15(2): 151-156.

Robinson BW, Creaney J, Lake R, Nowak A, Musk AW, de Klerk N, Winzell P, Hellstrom KE, Hellstrom I (2003) Mesothelin-family proteins and diagnosis of mesothelioma. Lancet 362(9396): 1612-1616.

Schneider J, Hoffmann H, Dienemann H, Herth FJ, Meister M, Muley T (2008) Diagnostic and prognostic value of soluble mesothelin-related proteins in patients with malignant pleural mesothelioma in comparison with benign asbestosis and lung cancer. J Thorac Oncol 3(11): 1317-1324.

Schouwink H, Korse CM, Bonfrer JM, Hart AA, Baas P (1999) Prognostic value of the serum tumour markers Cyfra 21-1 and tissue polypeptide antigen in malignant mesothelioma. Lung Cancer 25(1): 25-32.

Shojaei F, Scott N, Kang X, Lappin PB, Fitzgerald AA, Karlicek S, Simmons BH, Wu A, Lee JH, Bergqvist S, Kraynov E (2012) Osteopontin induces growth of metastatic tumors in a preclinical model of non-small lung cancer. J Exp Clin Cancer Res 31: 26.

Strizzi L, Catalano A, Vianale G, Orecchia S, Casalini A, Tassi G, Puntoni R, Mutti L, Procopio A (2001) Vascular endothelial growth factor is an autocrine growth factor in human malignant mesothelioma. J Pathol 193(4): 468-475.

Tabata C, Shibata E, Tabata R, Kanemura S, Mikami K, Nogi Y, Masachika E, Nishizaki T, Nakano T (2013) Serum HMGB1 as a prognostic marker for malignant pleural mesothelioma. BMC Cancer 13: 205.

Tajima K, Hirama M, Shiomi K, Ishiwata T, Yoshioka M, Iwase A, Iwakami S, Yamazaki M, Toba M, Tobino K, Sugano K, Ichikawa M, Hagiwara Y, Takahashi K, Hino O (2008) ERC/mesothelin as a marker for chemotherapeutic response in patients with mesothelioma. Anticancer Res 28(6B): 3933-3936.

Thylen A, Hjerpe A, Martensson G (2001) Hyaluronan content in pleural fluid as a prognostic factor in patients with malignant pleural mesothelioma. Cancer 92(5): 1224-1230.

Thylen A, Wallin J, Martensson G (1999) Hyaluronan in serum as an indicator of progressive disease in hyaluronan-producing malignant mesothelioma. Cancer 86(10): 2000-2005.

Vogelzang NJ, Rusthoven JJ, Symanowski J, Denham C, Kaukel E, Ruffie P, Gatzemeier U, Boyer M, Emri S, Manegold C, Niyikiza C, Paoletti P (2003) Phase III study of pemetrexed in combination with cisplatin $v s$ cisplatin alone in patients with malignant pleural mesothelioma. J Clin Oncol 21(14): 2636-2644.

Wheatley-Price P, Yang B, Patsios D, Patel D, Ma C, Xu W, Leighl N, Feld R, Cho BC, O'Sullivan B, Roberts H, Tsao MS, Tammemagi M, Anraku M, Chen Z, de Perrot M, Liu G (2010) Soluble mesothelin-related Peptide and osteopontin as markers of response in malignant mesothelioma. J Clin Oncol 28(20): 3316-3322.

Yamada S, Tabata C, Tabata R, Fukuoka K, Nakano T (2011) Clinical significance of pleural effusion mesothelin in malignant pleural mesothelioma. Clin Chem Lab Med 49(10): 1721-1726.

Yasumitsu A, Tabata C, Tabata R, Hirayama N, Murakami A, Yamada S, Terada T, Iida S, Tamura K, Fukuoka K, Kuribayashi K, Nakano T (2010) 
Clinical significance of serum vascular endothelial growth factor in malignant pleural mesothelioma. J Thorac Oncol 5(4): 479-483.

Zalcman G, Mazieres J, Margery J, Greillier L, Audigier-Valette C, Moro-Sibilot D, Molinier O, Corre R, Monnet I, Gounant V, Riviere F, Janicot H, Gervais R, Locher C, Milleron B, Tran Q, Lebitasy MP, Morin F, Creveuil C, Parienti JJ, Scherpereel A. French Cooperative Thoracic I (2016) Bevacizumab for newly diagnosed pleural mesothelioma in the mesothelioma avastin cisplatin pemetrexed study (MAPS): a randomised, controlled, open-label, phase 3 trial. Lancet 387(10026): 1405-1414.

This work is published under the standard license to publish agreement. After 12 months the work will become freely available and the license terms will switch to a Creative Commons AttributionNonCommercial-Share Alike 4.0 Unported License.

Supplementary Information accompanies this paper on British Journal of Cancer website (http://www.nature.com/bjc) 